\title{
Nuclear charge radii and electromagnetic moments of radioactive scandium isotopes and isomers
}

\author{
M Avgoulea ${ }^{1}$, Yu P Gangrsky ${ }^{2}, \mathrm{~K}$ P Marinova ${ }^{2}$, \\ S G Zemlyanoi ${ }^{2}$, S Fritzsche ${ }^{3,4}$, D Iablonskyi ${ }^{4}$, C Barbieri ${ }^{5}$, \\ E C Simpson ${ }^{5}$, P D Stevenson ${ }^{5}$, J Billowes ${ }^{1}$, P Campbell ${ }^{1}$, \\ B Cheal ${ }^{1}$, B Tordoff ${ }^{1}$, M L Bissell ${ }^{6}$, D H Forest ${ }^{6}$, \\ M D Gardner ${ }^{6}$, G Tungate ${ }^{6}$, J Huikari ${ }^{7}$, A Nieminen ${ }^{7}$, \\ H Penttilä ${ }^{7}$ and J Äystö ${ }^{7}$ \\ 1 School of Physics and Astronomy, University of Manchester, M13 9PL, UK \\ 2 FLNR Joint Institute for Nuclear Research, 141980 Dubna, Moscow Region, Russia \\ 3 GSI Helmholtzzentrum für Schwerionenforschung, 64291 Darmstadt, Germany \\ ${ }^{4}$ Department of Physics, University of Oulu, Fin-90014 Oulu, Finland \\ ${ }^{5}$ Department of Physics, University of Surrey, GU2 7XH, UK \\ 6 School of Physics and Astronomy, University of Birmingham, B15 2TT, UK \\ 7 Department of Physics, University of Jyväskylä, PB 35 (YFL) FIN-40351 \\ Jyväskylä, Finland
}

\begin{abstract}
Collinear laser spectroscopy experiments with the $\mathrm{Sc}^{+}$transition $3 d 4 s{ }^{3} D_{2} \rightarrow 3 d 4 p{ }^{3} F_{3}$ at $\lambda=363.1 \mathrm{~nm}$ were performed on the ${ }^{42-46} \mathrm{Sc}$ isotopic chain using an ion guide isotope separator with a cooler-buncher. Nuclear magnetic dipole and electric quadrupole moments as well as isotope shifts were determined from the hyperfine structure for five ground states and two isomers. Extensive multi-configurational Dirac-Fock calculations were performed in order to evaluate the specific mass-shift, $M_{\mathrm{SMS}}$, and field-shift, $F$, parameters which allowed evaluation of the charge radii trend of the Sc isotopic sequence. The charge radii obtained show systematics more like the $\mathrm{Ti}$ radii, which increase towards the neutron shell closure $N=20$, than the symmetric parabolic curve for Ca. The changes in mean square charge radii of the isomeric states relative to the ground states for ${ }^{44} \mathrm{Sc}$ and ${ }^{45} \mathrm{Sc}$ were also extracted. The charge radii difference between the ground and isomeric states of ${ }^{45} \mathrm{Sc}$ is in agreement with the deformation effect estimated from the $B(\mathrm{E} 2)$ measurements but is smaller than the deformation extracted from the spectroscopic quadrupole moments.
\end{abstract}

Keywords: collinear laser spectroscopy, mean-square charge radius, nuclear moments PACS numbers: 21.10.Ft, 21.10.Ky, 32.10.Fn, 42.62.Fi 


\section{Introduction}

The scandium isotopes $(Z=21)$ investigated in this study lie between the $Z, N=20$ and $Z, N=28$ shell closures. The trends (isotopic and isotonic) of the mean square (ms) charge radii of nuclei in this region are strongly influenced by several proton and neutron shell closures. Data on the charge radii trend for four isotopic chains in this region are already available covering the whole neutron $f_{7 / 2}$ shell (calcium $[1,2,3]$ and potassium [4]) or part of it (argon [5, 6] and titanium [7]) and even extending beyond it $(\mathrm{Ar}, \mathrm{K}$ and $\mathrm{Ca}$ ). These are displayed in figure 1. For the Ca isotopes across the $\nu\left(1 f_{7 / 2}\right)$ shell, the charge radii are characterized by a pronounced symmetric parabolic shape superimposed by a large odd-even staggering (OES) $[1,8]$. This symmetry is not reproduced by the radii behaviour of the neighbouring elements. The shape asymmetry of the $\delta\left\langle r^{2}\right\rangle$ curves for the elements with $Z>20$ and $Z<20$ occurs in opposite directions: for the $\mathrm{Ti}$ chain $(Z=22)$ there is a steady increase of the charge radii towards $N=20$ [7], whereas for the chains with $Z=18,19$ the radii increase towards $N=28[4,6]$. The OES effect decreases away from calcium, to argon or titanium, but is smallest for the odd $-Z$ element potassium. Systematic measurements of the isotopes below the $N=20$ shell closure have only been made for the Ar chain and show no shell effect at $N=20[5,6]$. In the $\mathrm{Ca}$ and $\mathrm{K}$ chains the sequential addition of neutrons going from the $s d$ - to the $f_{7 / 2}$-shells, similarly gives a smooth change of the successive ms charge radii for the odd $-N$ isotopes. This behaviour is in contrast with what is expected from the global nuclear radii behaviour at neutron shell closures with $N \geq 28$ [9].

Several descriptions exist $[5,10,11]$ which are able to explain some of the observed features. Nevertheless, there remain many open questions, especially about the influence of the closed proton shell of Ca. It should be clarified whether the flattening of the curves away from $\mathrm{Ca}$ is due to the increasing distance from $Z=20$ or can be ascribed to a pairing effect (odd $-Z /$ even $-Z$ ) and whether the absence of a shell effect at $N=20$ will persist in the charge radii evolution of elements with $Z>20$. A better understanding of the charge radii peculiarities in the calcium region requires extension of the experimental information. Of particular importance is scandium $(Z=21)$, one of the odd $-Z$ neighbours of $\mathrm{Ca}$. Information on nuclear charge radii in the scandium isotopic sequence will provide a clearer picture about the role of the proton pairing effect. No charge radii measurements have been made prior to this work with the exception of the stable ${ }^{45} \mathrm{Sc}$ ground state.

Magnetic dipole and electric quadrupole moments of nuclear ground and isomeric states can be obtained from measurements of the hyperfine structures [12]. A specific example of interest in this context is ${ }^{45} \mathrm{Sc}$ which-along with ${ }^{43} \mathrm{Ca}$ and ${ }^{45} \mathrm{Ti}$ - is considered as an excellent example of shape coexistence between the spherical $7 / 2^{-}$ ground state and deformed $3 / 2^{+}$isomeric structures $[13,14]$. All of these provide a discerning testing ground for microscopic model calculations. Nuclear moments of Sc nuclei known prior to this work are summarised in reference [15].

Low production yields of scandium isotopes at conventional isotope separators, have 


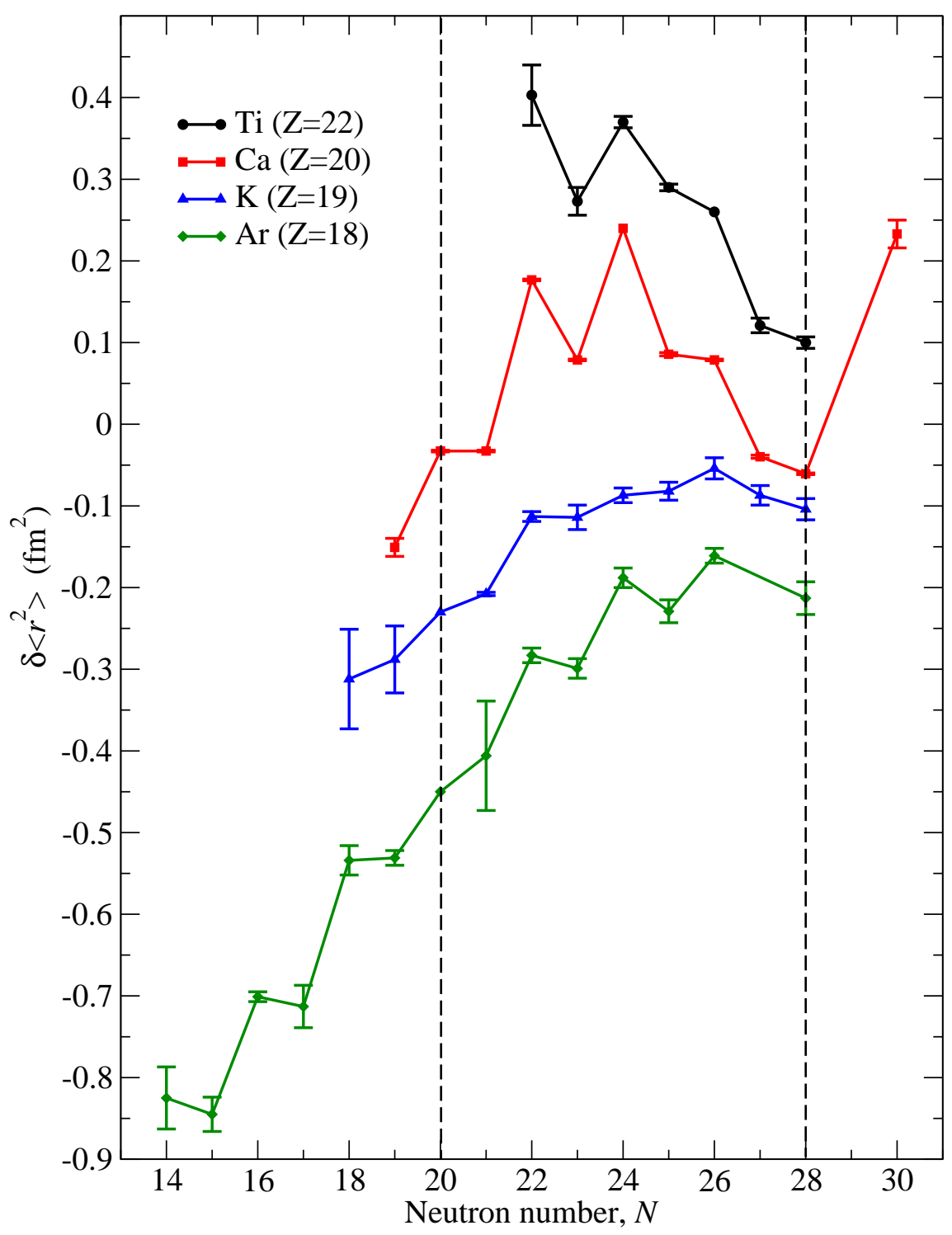

Figure 1. Changes in ms charge radii versus neutron number, $N$, for the Ti, Ca, K and Ar chains. Dotted lines indicate neutron shell gaps. The isotope chains have been offset from one another for clarity.

restricted their study so far. This paper reports the first collinear laser spectroscopy results for the radioactive scandium isotopes, ${ }^{42-44,46} \mathrm{Sc}$, including the ${ }^{44 \mathrm{~m}} \mathrm{Sc}\left(I=6^{+}\right)$ and ${ }^{45 \mathrm{~m} S c}\left(I=3 / 2^{+}\right)$isomers. Our earlier published data [16] were preliminary and did not include the atomic calculations required to determine the ms charge radii from the isotope shifts. The experimental work was undertaken at the University of Jyväskylä, Finland where the pioneering development of the IGISOL (ion-guide isotope separator on-line) facility [17] has enabled the production of radioactive beams of scandium isotopes with sufficient yields for laser spectroscopy experiments. A substantial improvement in the quality of the produced ion beams was achieved following the installation of a cooler-buncher $[18,19,20]$ in the beam line, thus increasing the 
sensitivity of the collinear beams method of laser fluorescence spectroscopy.

\section{Experimental method}

\subsection{Laser spectroscopy of the stable ${ }^{45} \mathrm{Sc}$ isotope}

Preliminary off-line studies were carried out at the IGISOL facility to optimize the spectroscopy. Stable $\mathrm{Sc}^{+}$beam currents of $\sim 20 \mathrm{pA}$ were produced from a scandium sample at the cathode of a $\sim 500 \mathrm{~V}$ discharge source inside the IGISOL chamber. Four $\mathrm{Sc}^{+}$transitions were chosen to be studied on the grounds of their oscillator strength. The ions were accelerated to $37 \mathrm{keV}$ and Doppler tuned onto resonance with the frequency doubled output of a laser locked and stabilized to a chosen molecular iodine absorption line. The wavelengths and upper and lower hyperfine structure parameters: $A_{\mathrm{u}}, B_{\mathrm{u}}, A_{\mathrm{l}}$ and $B_{1}$, of the four transitions are presented in table 1 . The transition $3 d 4 s^{3} D_{2}(67$ $\left.\mathrm{cm}^{-1}\right) \rightarrow 3 d 4 p^{3} F_{3}\left(27602 \mathrm{~cm}^{-1}\right)$ at $363.1 \mathrm{~nm}$ was chosen for the on-line experiment, due to the better spectroscopic efficiency, sensitivity to the upper and lower state $B$ hyperfine parameters and also because its structure does not extend in frequency space as much as the structures of the other three lines as illustrated in figure 2.

Table 1. Upper and lower level hyperfine parameters along with spectroscopic efficiencies (for the strongest hyperfine component) of the four ionic transitions investigated for the stable ${ }^{45} \mathrm{Sc}$ isotope.

\begin{tabular}{llllllll}
\hline$\lambda$ & $\begin{array}{l}\text { Lower } \\
\text { level }\end{array}$ & $\begin{array}{l}\text { Upper } \\
\text { level }\end{array}$ & $\begin{array}{l}A_{\mathrm{l}} \\
(\mathrm{MHz})\end{array}$ & $\begin{array}{l}B_{\mathrm{l}} \\
(\mathrm{MHz})\end{array}$ & $\begin{array}{l}A_{\mathrm{u}} \\
(\mathrm{MHz})\end{array}$ & $\begin{array}{l}B_{\mathrm{u}} \\
(\mathrm{MHz})\end{array}$ & $\begin{array}{l}\text { Efficiency } \\
\text { (photon/ion) }\end{array}$ \\
\hline 364.3 & ${ }^{3} D_{1}$ & ${ }^{3} F_{2}^{\mathrm{o}}$ & $-479.9(5)$ & $-12.6(19)$ & $+368.3(3)$ & $-61.7(32)$ & $1 / 29,000$ \\
358.1 & ${ }^{3} D_{1}$ & ${ }^{3} D_{1}^{\mathrm{o}}$ & $-479.9(5)$ & $-17.6(37)$ & $+305.3(6)$ & $+16.3(36)$ & $1 / 150,000$ \\
363.1 & ${ }^{3} D_{2}$ & ${ }^{3} F_{3}^{\circ}$ & $+507.9(1)$ & $-34.4(15)$ & $+205.7(1)$ & $-62.3(19)$ & $1 / 27,000$ \\
361.4 & ${ }^{3} D_{3}$ & ${ }^{3} F_{4}^{\circ}$ & $+656.2(6)$ & $-43(14)$ & $+101.5(5)$ & $-81(14)$ & $1 / 25,000$ \\
\hline
\end{tabular}

\subsection{Measurements of radioactive scandium isotopes and isomers}

Radioactive scandium isotopes were produced at the IGISOL facility by proton and deuteron beam irradiation of a $2.1 \mathrm{mg} \cdot \mathrm{cm}^{-2}{ }^{45} \mathrm{Sc}$ target. A deuteron beam energy of $15 \mathrm{MeV}$ and proton beam energies of $25-48 \mathrm{MeV}$ with currents of $5-10 \mu \mathrm{A}$ were used for the production of ${ }^{42,43,44,44 \mathrm{~m}, 45,45 \mathrm{~m}, 46} \mathrm{Sc}$ via (p,p $\left.x \mathrm{n}\right)$ and (d,p) reactions. The nuclear reaction products were thermalized in the IGISOL in a fast flowing jet of helium gas at pressures of $60-230$ mbar and were then extracted and mass separated. A radiofrequency gas-filled quadrupole (on a high voltage platform $100 \mathrm{~V}$ below the IGISOL potential) was used to cool the singly charged ion beam to reduce its energy spread to $<1 \mathrm{eV}$ [18]. The ion beam was then reaccelerated by the platform high voltage and overlapped collinearly at the interaction region with $0.5 \mathrm{~mW}$ of laser light with 


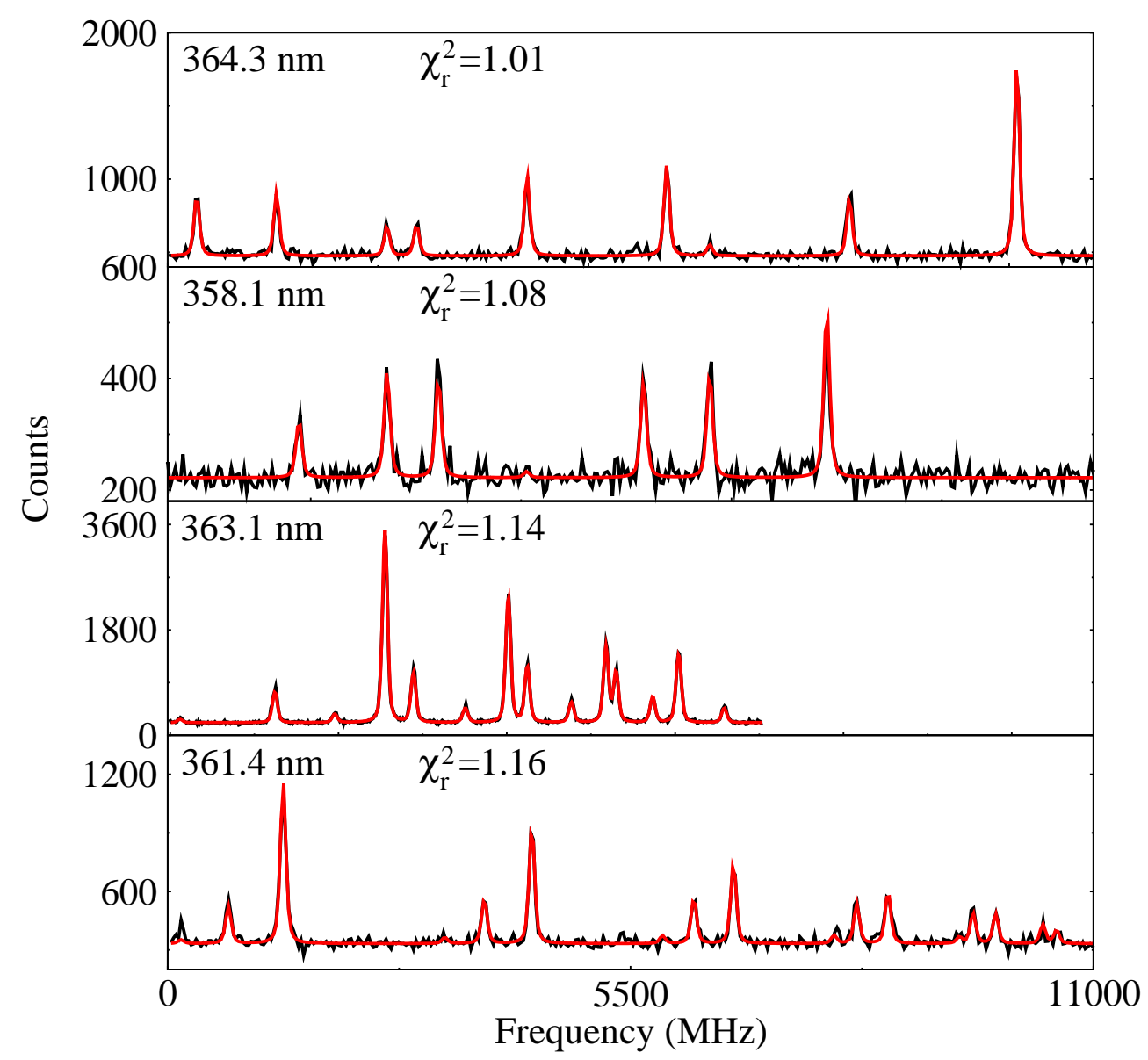

Figure 2. Hyperfine structures of the $364.3 \mathrm{~nm}, 358.1 \mathrm{~nm}, 363.1 \mathrm{~nm}$ and $361.4 \mathrm{~nm}$ lines for the stable ${ }^{45} \mathrm{Sc}$ isotope investigated during the off-line preparation tests. Fitted Voigt profiles are also shown.

$\lambda=363.1 \mathrm{~nm}$. This UV wavelength was generated by intra-cavity frequency doubling using a $\mathrm{LiIO}_{3}$ crystal in a Spectra Physics 380D dye laser running with Pyridine 2 dye. The fundamental laser frequency was stabilized to within $\sim 2 \mathrm{MHz}$ and locked to the reference frequency of a molecular iodine absorption line at $13748.191 \mathrm{~cm}^{-1}$. An adjustable potential was applied to the laser-ion interaction region to Doppler tune the ions onto resonance with the laser beam and the fluorescent photons were imaged through a system of lenses onto a Hamamatsu R5900-P03-L16 photomultiplier tube. Ions were accumulated and bunched in the cooler-buncher over a 100 ms cycle. Typically and ion bunch contains less than $\sim 10^{5}$ ions including any isobaric contaminants. A reduction of scattered, non-resonant laser light by a factor of $1.4 \times 10^{-4}$ was achieved by electronically gating the photomultiplier signal for $15 \mu$ s such that photon events were only accepted if they arrived when an ion bunch was in front of the detector [19, 20]. Scans of radioisotopes were alternated with scans of ${ }^{45} \mathrm{Sc}$ naturally sputtered from the target which provided an on-line test for the stability of the laser frequency and the accelerating potential, with the differential error on the latter found to be within $0.1 \mathrm{~V}$. 
Table 2. Upper state hyperfine parameters of the ${ }^{43-46,44 \mathrm{~m}, 45 \mathrm{~m}} \mathrm{Sc}$ isotopes and isomers measured on the $363.1 \mathrm{~nm}$ ionic transition.

\begin{tabular}{llll}
\hline$A$ & $I^{\pi}$ & $A_{\mathrm{u}}(\mathrm{MHz})$ & $B_{\mathrm{u}}(\mathrm{MHz})$ \\
\hline 43 & $7 / 2^{-}$ & $+195.8(4)$ & $-77(15)$ \\
44 & $2^{+}$ & $+189.1(4)$ & $+44(11)$ \\
$44 \mathrm{~m}$ & $6^{+}$ & $+96.7(3)$ & $-58(24)$ \\
45 & $7 / 2^{-}$ & $+205.7(1)$ & $-62.3(19)$ \\
$45 \mathrm{~m}$ & $3 / 2^{+}$ & $+36.3(11)$ & $+78(15)$ \\
46 & $4^{+}$ & $+115.1(3)$ & $+35(4)$ \\
\hline
\end{tabular}

\section{Analysis and results}

\subsection{Hyperfine structure and electromagnetic moments}

For each scandium isotope several spectra were recorded at the same acceleration energy and were summed. An example of the summed resonance spectra, converted to frequency relative to the centroid of ${ }^{45} \mathrm{Sc}$, is shown in figure 3 . The resonance peak positions were established by fitting the data with a hyperfine structure composed of Voigt profiles, which were found to describe most adequately the line shape. The magnetic dipole and electric quadrupole coupling constants $A$ and $B$ of both lower, $3 d 4 s^{3} D_{2}$, and upper, $3 d 4 p^{3} F_{3}$, states were obtained from the experimental spectra using a least square fitting procedure to the two-parameter first-order hyperfine splitting formula [21],

$$
\Delta \nu_{F}=A \frac{C}{2}+B \frac{3 C(C+1)-4 I(I+1) J(J+1)}{8 I(2 I-1) J(2 J-1)},
$$

where $C=F(F+1)-I(I+1)-J(J+1)$ and $I, J, F$ are the nuclear, electronic and total atomic angular momentum quantum numbers, respectively. The number of observed or resolved lines in some cases was not sufficient to extract all of the $A$ and $B$ factors of the lower and upper level independently. For this reason, the lower state hyperfine parameters $A_{1}, B_{1}$ were scaled for all isotopes to the ratios of the $A$ and $B$ parameters observed in the ${ }^{45} \mathrm{Sc}$ ground state $\left(A_{\mathrm{l}} / A_{\mathrm{u}}=+2.4686(13)\right.$ and $\left.B_{\mathrm{l}} / B_{\mathrm{u}}=+0.552(29)\right)$. The hyperfine anomaly is negligible relative to our experimental uncertainties (see e.g. [22]) and was therefore neglected in the analysis. The peak intensity ratios in the fit were fixed to the expected hyperfine intensities as calculated from the $6 \mathrm{~J}$ symbols.

Values of the nuclear moments - magnetic dipole $(\mu)$ and electric quadrupole $\left(Q_{s}\right)$ were deduced from the $A$ and $B$ factors with reference to the highly accurate nuclear moments of the stable ${ }^{45} \mathrm{Sc}$ isotope (see reference [15] and the references therein) according to the relations,

$$
\mu_{1}=\frac{A_{1} I_{1}}{A_{2} I_{2}} \mu_{2} \quad \text { and } \quad Q_{s 1}=\frac{B_{1}}{B_{2}} Q_{s 2} .
$$

The moments derived from relations (2) are displayed in table 3. Their values are in reasonable agreement with those published in the compilation of Stone [15] but provide 


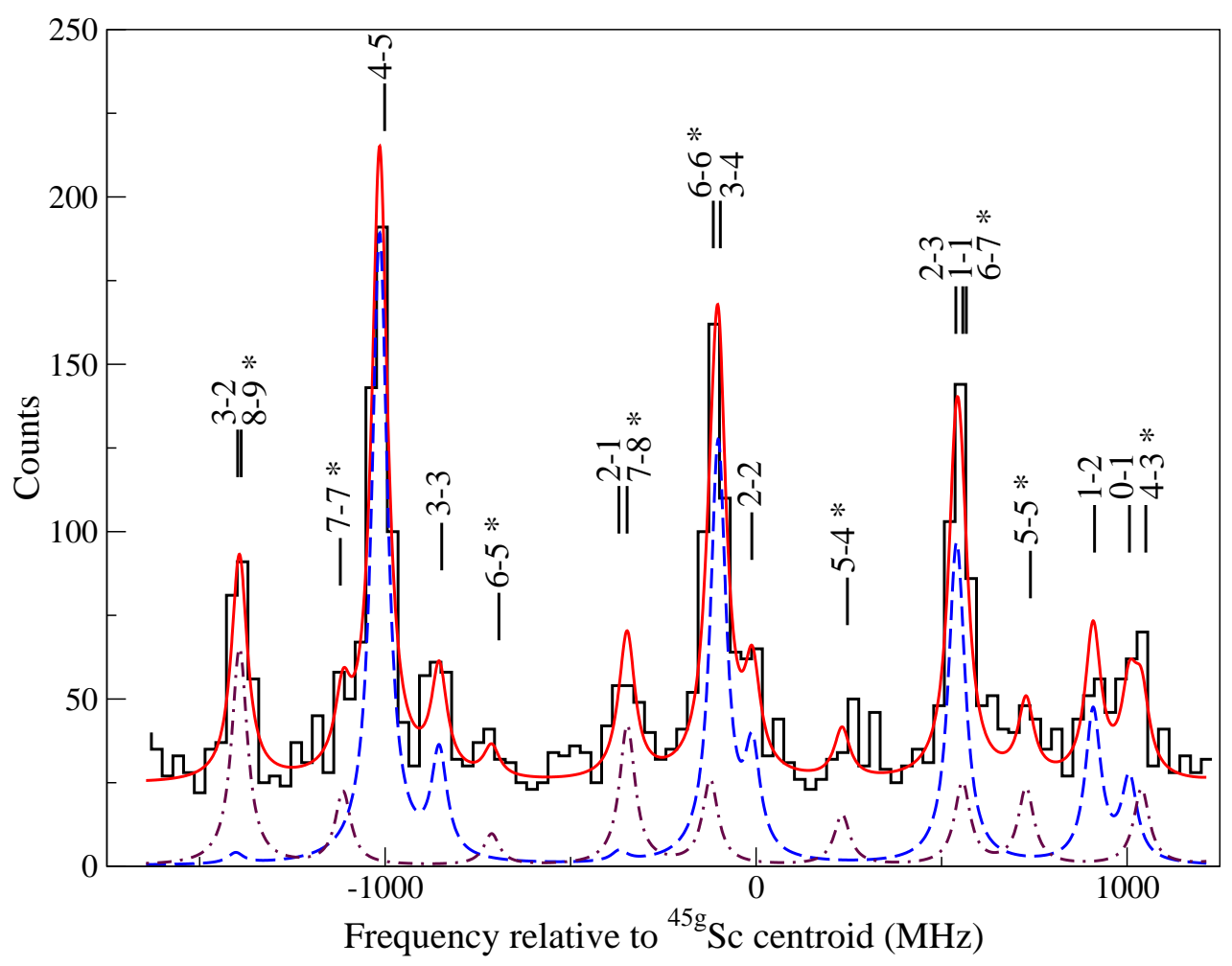

Figure 3. Example of a resonance fluorescence spectrum for ${ }^{44 \mathrm{~g}, \mathrm{~m}} \mathrm{Sc}$. The fitted structure is shown overlaid, with the separate ground state (dashed) and isomeric (dot-dashed) components underneath. Hyperfine $F_{1} \rightarrow F_{\mathrm{u}}$ transitions are indicated, with isomeric components denoted with an asterisk $\left(^{*}\right)$. Other spectra (not shown) with overlapping scan regions were also taken and analyzed simultaneously. Spectra for the other isotopes is contained in reference [16].

Table 3. Nuclear moments of the ${ }^{43-46,44 \mathrm{~m}, 45 \mathrm{~m}}$ Sc isotopes and isomers determined from this work along with those from the compilation of Stone [15].

\begin{tabular}{llllll}
\hline$A$ & $I^{\pi}$ & $\begin{array}{l}\mu\left(\mu_{\mathrm{N}}\right) \\
\text { this work }\end{array}$ & $\begin{array}{l}\mu\left(\mu_{\mathrm{N}}\right) \\
\text { Ref. [15] }\end{array}$ & $\begin{array}{l}Q_{s}(\mathrm{~b}) \\
\text { this work }\end{array}$ & $\begin{array}{l}Q_{s}(\mathrm{~b}) \\
\text { Ref. [15] }\end{array}$ \\
\hline 43 & $7 / 2^{-}$ & $+4.528(10)$ & $+4.62(4)$ & $-0.27(5)$ & $-0.26(6)$ \\
44 & $2^{+}$ & $+2.499(5)$ & $+2.56(3)$ & $+0.16(4)$ & $+0.10(5)$ \\
$44 \mathrm{~m}$ & $6^{+}$ & $+3.833(12)$ & $+3.88(1)$ & $-0.21(9)$ & $-0.19(2)$ \\
45 & $7 / 2^{-}$ & [reference] & $+4.756487(2)$ & [reference] & $-0.220(2)$ \\
$45 \mathrm{~m}$ & $3 / 2^{+}$ & $+0.360(11)$ & - & $+0.28(5)$ & - \\
46 & $4^{+}$ & $+3.042(8)$ & $+3.03(2)$ & $+0.12(2)$ & $+0.119(6)$ \\
\hline
\end{tabular}

a higher precision for the magnetic moment. Both of the nuclear moments for the isomer ${ }^{45 \mathrm{~m}} \mathrm{Sc}$ are deduced for the first time.

\subsection{Isotope shifts}

The offset in centroid frequency between the hyperfine structures of two nuclear states, $\nu^{A, A^{\prime}}=\nu^{A^{\prime}}-\nu^{A}$, is known as the isotope (or isomer) shift. All isotope and isomer shifts 
have been obtained for the first time. The isotope shift can be decomposed into the field-shift (FS) and two terms accounting for the finite mass of the nucleus: the normal and specific mass-shifts (NMS and SMS) where [21, 23],

$$
\begin{aligned}
\delta \nu^{45, A} & =\delta \nu_{\mathrm{FS}}^{45, A}+\delta \nu_{\mathrm{NMS}}^{45, A}+\delta \nu_{\mathrm{SMS}}^{45, A} \\
& =F \delta\left\langle r^{2}\right\rangle^{45, A}+\frac{m_{A}-m_{45}}{m_{A} m_{45}}\left(M_{\mathrm{NMS}}+M_{\mathrm{SMS}}\right) .
\end{aligned}
$$

Here, $M_{\mathrm{NMS}}$ and $M_{\mathrm{SMS}}$ are the normal and specific mass-shift constants, respectively, $F$ is the electronic factor related to the change in the electronic density at the nucleus for the optical transition and $m_{A}$ is the mass of the isotope with atomic number $A$.

Traditionally, $F$ has been evaluated from atomic electron shell data using either semi-empirical procedures and/or Hartree-Fock methods for calculating the relevant electronic density at the site of the nucleus. So far these evaluations have yielded very consistent sets of $\delta\left\langle r^{2}\right\rangle$ values throughout the nuclear chart, including a number of very long isotopic chains [8]. The electronic factor $F$ is given by

$$
F=\pi a_{0}^{3} \frac{\Delta|\Psi(0)|^{2}}{Z} f(Z)
$$

where $a_{0}$ is the Bohr radius and the total electron density change can be written

$$
\Delta|\Psi(0)|^{2}=\beta|\Psi(0)|_{n s}^{2} .
$$

The $\Delta|\Psi(0)|^{2}$ in equation 4 represents the non-relativistic change in electron density at the nucleus between lower and upper states of the optical transition and $f(Z)$ is a relativistic atomic factor tabulated in $[24,25,26]$. The electron density, $|\Psi(0)|_{n s}^{2}$ is for a single $n s$ electron and $\beta$ is a factor accounting for the screening of inner closed-shell electrons from the nuclear charge by the valence electrons.

As regards the mass-shift, the situation is considerably more complicated. The normal mass-shift constant, given by $M_{\mathrm{NMS}}=\nu m_{\mathrm{e}}=+452.8 \mathrm{GHz} \cdot \mathrm{u}$, is calculated with the transition frequency $\nu$ and the electron mass $m_{\mathrm{e}}$. The specific mass-shift constant, $M_{\mathrm{SMS}}$, accounting for correlations of the electron motion, is much more difficult to calculate reliably. Unfortunately, scandium has only a single stable isotope and there are no other experimental charge radii data which would allow a determination of the specific mass-shift in a consistent way. The often used approximate technique of a King plot [23] between the isotope shifts of one element versus the ms charge radii of a neighbouring isotopic chain $[27,28,29]$ is impossible in the case of Sc due to the lack of correspondence between neighbouring chains.

\subsection{Calculation of the specific mass-shift and field-shift parameters}

A more rigorous treatment of the specific mass-shift and field-shift parameters, $M_{\mathrm{SMS}}$ and $F$, is obtained if the electronic structure of the atom is described as a many-electron system. Especially for open-shell atoms and ions, the multi-configuration Dirac-Fock (MCDF) method has been found to be a versatile tool to calculate and analyze many different properties of such systems, from tiny-to-small level shifts due to the structure 
of the nucleus, e.g. the hyperfine and isotope shifts [30], up to the ionization and recombination of atoms following their interaction with external particles and fields [31]. Apart from a rather systematic treatment of the wave functions of atomic bound states, the MCDF method enables one to deal on equal footings with the effects of relativity and many-electron correlations.

The MCDF method has been described in detail in the literature [32]. In this method, an atomic state is approximated by a linear combination of so-called configuration state functions (CSF) of the same symmetry

$$
\psi_{\alpha}(P J M)=\sum_{r=1}^{n_{c}} c_{r}(\alpha)\left|\gamma_{r} P J M\right\rangle
$$

where $n_{c}$ is the number of CSFs and $\left\{c_{r}(\alpha)\right\}$ denotes the representation of the atomic state in this basis. In most standard computations, the CSFs $\left|\gamma_{r} P J M\right\rangle$ are constructed as antisymmetrized products of a common set of orthonormal orbitals and are optimized together on the basis of the Dirac-Coulomb Hamiltonian. Relativistic effects due to the Breit interaction are then added to the representation $\left\{c_{r}(\alpha)\right\}$ by diagonalizing the Dirac-Coulomb-Breit Hamiltonian matrix [33, 34]. The dominant QED corrections can also be estimated within this method as well but are negligible for optical transitions of mid- $Z$ elements. In addition, the specific mass-shift (operator) can be taken into account into the Hamiltonian matrix as implemented, for example, within the RELCI code $[35,36]$. The field-shift due to different charge distributions of the isotopes were taken into account by means of an extended nucleus with a two-parameter Fermi distribution

$$
\rho(r)=\frac{\rho_{o}}{1+e^{(r-c) / a}}
$$

where $c$ is the 'half-charge density' radius and $a$ characterizes the skin thickness. Since we only aim for the field-shift parameter $F$, a spherical symmetric nucleus was assumed for all isotopes, and the Fermi parameters were taken directly from Grasp92 [33].

Since the isotope shift depends on the details of the wave functions near to the nucleus (cf. equation (4)), special care has to be taken for the generation of the atomic states of interest, including not only valence-valence correlations but also the polarization of the core and even core-core correlations (as far as possible). The importance of these different classes of electronic correlations was shown for the isotope shift of the optical $4 s^{2} S_{1 / 2}-4 p^{2} P_{1 / 2,3 / 2}$ resonance transitions of singly-charged $\mathrm{Ca}^{+}$ ions by applying many-body perturbation theory [37]. In the MCDF method, these correlations are taken into account similarly by including systematically single and double (and possibly further) replacements of electrons from the bound into virtual orbitals. Using such a 'shell-model' procedure, however, the size of the wave function expansion, $n_{c}$, often increases very rapidly and makes it necessary to first identify the major correlations, and to restrict the computations accordingly. For a singly-charged ion with an open $d$-shell, such as $\mathrm{Sc}^{+}$, this usually implies a restriction to two layers of additional (virtual) correlation orbitals as well as a proper selection of the corecore correlations that can be considered. Large wave function expansions of tens or 
hundreds of thousands of CSFs are feasible today and allow for reasonably correlated level calculations even for nearly-neutral atoms with open $d$ - and $f$-shells $[30,38]$.

To obtain the mass- and field-shift parameters for the $3 d 4 s{ }^{3} D_{2} \rightarrow 3 d 4 p{ }^{3} F_{3}$ transition in $\mathrm{Sc}^{+}$, a series of computations have been performed, based on the $3 p^{6} 3 d^{2}, \quad 3 p^{6} 3 d 4 s, \quad 3 p^{6} 4 s^{2}, \quad 3 p^{6} 4 p^{2}$ even-parity and $3 p^{6} 3 d 4 p$ odd-parity reference configurations (all with a $1 s^{2} 2 s^{2} 2 p^{6} 3 s^{2} 3 p^{6}$ fixed core). From these reference configurations, all single and double excitations into the $5 s, 5 p, 5 d$ and $5 f$ shells ( $5 l$ layer) as well as into the $6 s, 6 p, 6 d, 6 f$ shells (6l layer) have been incorporated successively, giving rise to maximal expansions $n_{c} \sim 60,000$. In addition, single excitations from the $1 s, \ldots, 4 p$ shells into the $5 l$ layer were taken into account to include the polarization of the core. While a computation with only the reference configuration included gives rise to $M_{\mathrm{SMS}}=-277 \mathrm{GHz} \cdot \mathrm{u}$ and $F=-274 \mathrm{MHz} \cdot \mathrm{fm}^{-2}$, and thus to a rather unphysical value of the specific mass-shift, the core-polarization to the reference configurations is enough to reverse the sign to $M_{\mathrm{SMS}}=+290 \mathrm{GHz} \cdot \mathrm{u}$. However, only a further stepwise increase of the size of the wave functions allows a reasonable convergence of the mass- and field-shift parameters to be monitored. Although no complete convergence (with regard to an arbitrary further increase in the size of the wave functions) could be obtained, we estimate from the various steps of the computations an uncertainty of about $25 \%$ for the specific mass-shift and $15 \%$ for the field-shift,

$$
\begin{aligned}
& M_{\mathrm{SMS}}=+130(30) \mathrm{GHz} \cdot \mathrm{u} \\
& F \quad=-355(50) \mathrm{MHz} \cdot \mathrm{fm}^{-2} .
\end{aligned}
$$

These 'uncertainties' were estimated from further test computations concerning different core-core correlations as well as the (incomplete) incorporation of a third correlation layer.

\subsection{Isotopic and isomeric charge radii changes}

The values of $\delta\left\langle r^{2}\right\rangle$ have been derived using the values for the electronic factor and specific mass-shift obtained in the previous section (see equations (8) and (9)). The data are compiled in table 4 and shown in figure 4 . An uncertainty on the specific mass shift of $25 \%$, dominates the error causing a pivoting about the reference isotope (see figure 4). The relatively small uncertainty on $F$ only scales the final values of $\delta\left\langle r^{2}\right\rangle$. As can be seen, the systematic errors affect only the overall scale of the charge radii and do not influence the relative effects between the isotopes. Note that ms charge radii changes between ground and isomeric states depend only on $F$ and are not affected by the uncertainty in $M_{\mathrm{SMS}}$. 
Table 4. Scandium isotope and isomer shifts, $\delta \nu^{A, A^{\prime}}=\nu^{A^{\prime}}-\nu^{A}$, measured on the $363.1 \mathrm{~nm}$ ionic transition, separate mass shift and field shift components, and the extracted ms charge radii, $\delta\left\langle r^{2}\right\rangle_{\exp }^{A, A^{\prime}}=\left\langle r^{2}\right\rangle^{A^{\prime}}-\left\langle r^{2}\right\rangle^{A}$. All ground state values are with respect to $A=45$, and isomeric state values are quoted with respect to the corresponding ground state. The errors quoted in parenthesis are statistical and those in square brackets represent the systematic errors arising from uncertainties in the scaling factors $F$ and $M_{\mathrm{SMS}}$. For comparison, $\delta\left\langle r^{2}\right\rangle_{\mathrm{SM}}^{A, A^{\prime}}$, from the shell-model calculations of this work are included.

\begin{tabular}{llllllll}
\hline$A$ & $A^{\prime}$ & $\delta \nu^{A, A^{\prime}}(\mathrm{MHz})$ & $\delta \nu_{\mathrm{MS}}^{A, A^{\prime}}$ & $\delta \nu_{\mathrm{FS}}^{A, A^{\prime}}$ & $\delta\left\langle r^{2}\right\rangle_{\exp }^{A, A^{\prime}}\left(\mathrm{fm}^{2}\right)$ & $\delta\left\langle r^{2}\right\rangle_{\mathrm{SM}}^{A, A^{\prime}}\left(\mathrm{fm}^{2}\right)$ \\
\hline 45 & 42 & $-985(11)$ & -924 & -61 & $+0.172(31)[136]$ & -0.076 \\
45 & 43 & $-631(5)$ & -602 & -29 & $+0.082(14)[88]$ & -0.026 \\
45 & 44 & $-287(4)$ & -294 & +7 & $-0.019(11)[43]$ & -0.014 \\
44 & $44 \mathrm{~m}$ & $+25(4)$ & 0 & +25 & $-0.070(11)[10]$ & -0.041 & \\
45 & 45 & 0 & 0 & 0 & 0 & 0 \\
45 & $45 \mathrm{~m}$ & $-66(2)$ & 0 & -66 & $+0.186(6)[26]$ & +0.068 \\
45 & 46 & $+336(3)$ & +282 & +54 & $-0.152(8)[46]$ & -0.016 \\
\hline
\end{tabular}

\section{Discussion}

\subsection{Nuclear charge radii}

4.1.1. Radii changes and deformation of ${ }^{45 \mathrm{~g}, \mathrm{~m}} \mathrm{Sc}$.

Among the investigated nuclei, ${ }^{45} \mathrm{Sc}$ deserves closer attention. It belongs to the several odd $-A$ nuclei from the lower $f_{7 / 2}$ shell, e.g. ${ }^{43} \mathrm{Ca},{ }^{43,45,49} \mathrm{Sc},{ }^{45} \mathrm{Ti}$, and ${ }^{47,49} \mathrm{~V}$, showing evidence of collective behaviour as regular rotational-like bands are formed from their low lying positive-parity isomeric states, $I^{\pi}=3 / 2^{+}$. The latter result from the coupling of the valence $f p$ nucleons and particle-hole excitations across the $Z=N=20$ shell closure. The level schemes of ${ }^{45} \mathrm{Sc}$ have been studied experimentally [13, 14, 40, 41, 42] and theoretically $[43,44]$. It is suggested that the negative-parity ground state indicates a spherical structure, while a rotational-like band is formed upon the $I^{\pi}=3 / 2^{+}, 12 \mathrm{keV}$ intruder level in ${ }^{45 \mathrm{~m}} \mathrm{Sc}$. Therefore, the case of ${ }^{45} \mathrm{Sc}$ is a good example of shape coexistence of spherical and prolate-deformed structures.

The mean-square quadrupole deformation, $\left\langle\beta_{2}^{2}\right\rangle$, can be calculated using the reduced $B(\mathrm{E} 2)$ values between states of spin $I_{\mathrm{i}}$ and $I_{\mathrm{f}}$ (deduced from reference [13]) using the expression

$$
\left\langle\beta_{2}^{2}\right\rangle=\left(\frac{4 \pi}{5 Z e\left\langle r^{2}\right\rangle_{\mathrm{sph}}}\right)^{2} B\left(\mathrm{E} 2: I_{\mathrm{i}} \rightarrow I_{\mathrm{f}}\right) .
$$

The nuclear size is given most adequately by the droplet model [45] value of $\left\langle r^{2}\right\rangle_{\mathrm{sph}}=$ $12.366 \mathrm{fm}^{2}$ and is for a spherical nucleus of the same volume (calculated using the parameters of reference [46]). For the isomeric state, the deformation is averaged over the rotational band and $\left\langle\beta_{2}^{2}\right\rangle\left({ }^{45 \mathrm{~m}} \mathrm{Sc}\right)=0.052(4)$ is obtained for the ms quadrupole deformation. The ground state is approximately spherical and it is assumed that $\left\langle\beta_{2}^{2}\right\rangle\left({ }^{45 \mathrm{~g}} \mathrm{Sc}\right) \approx 0$. 


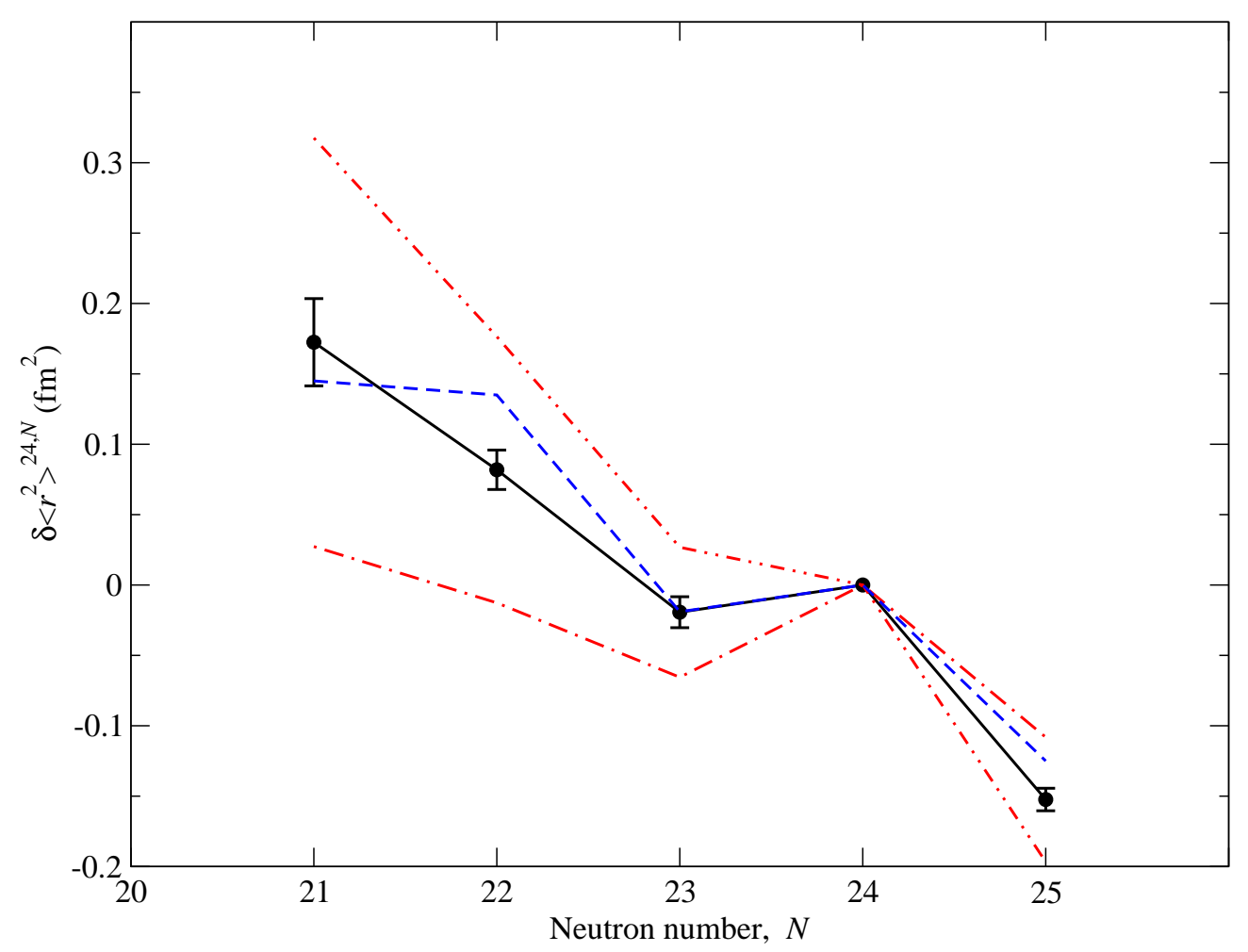

Figure 4. Neutron number dependence of the ${ }^{42-46} \mathrm{Sc}$ charge radii. Experimental data are denoted by full circles and the error bars represent statistical errors; the two enveloping lines indicate the effect of the specific mass shift uncertainties of $25 \%$. The dashed line represents a fit to the Zamick formula [39].

Using the relation,

$$
\delta\left\langle r^{2}\right\rangle^{\mathrm{g}, \mathrm{m}}=\left\langle r^{2}\right\rangle_{\mathrm{ss} . \mathrm{sph}} \frac{5}{4 \pi}\left[\left\langle\beta_{2}^{2}\right\rangle(45 \mathrm{~m})-\left\langle\beta_{2}^{2}\right\rangle(45)\right],
$$

where $\left\langle r^{2}\right\rangle_{\text {ss.sph }}=9.426 \mathrm{fm}^{2}$ [46] is for an equivalent sharp-surfaced spherical nucleus, an estimate of $\delta\left\langle r^{2}\right\rangle^{45 \mathrm{~g}, \mathrm{~m}}=+0.195(15) \mathrm{fm}^{2}$ can be made from the $B(\mathrm{E} 2)$ value. This compares favourably with the experimental value of $\delta\left\langle r^{2}\right\rangle^{45 \mathrm{~g}, \mathrm{~m}}=+0.186(26) \mathrm{fm}^{2}$.

An alternative approach is to use the $\left\langle\beta_{2}\right\rangle$ values derived from the spectroscopic quadrupole moments. For the well defined rotational band of ${ }^{45 \mathrm{~m}} \mathrm{Sc}$ we assume that the projection formula

$$
Q_{0}=\frac{(I+1)(2 I+3)}{I(2 I-1)} Q_{\mathrm{s}}
$$

is valid. With $Q_{\mathrm{s}}\left({ }^{45 \mathrm{~m}} \mathrm{Sc}\right)$ from table 3 , it follows that $Q_{0}\left({ }^{45 \mathrm{~m}} \mathrm{Sc}\right)=+1.38(27) \mathrm{b}$. Using the relation

$$
Q_{0} \approx \frac{5 Z\left\langle r^{2}\right\rangle_{\mathrm{sph}}}{\sqrt{5 \pi}}\left\langle\beta_{2}\right\rangle\left(1+0.36\left\langle\beta_{2}\right\rangle\right)
$$

the mean deformation is calculated as $\left\langle\beta_{2}\right\rangle\left({ }^{45 \mathrm{~m}} \mathrm{Sc}\right)=+0.374(73)$. If a similar attempt is made for the ground state, a value of $Q_{0}\left({ }^{45 \mathrm{~g}} \mathrm{Sc}\right)=-0.47(2)$ b may be deduced from the spectroscopic quadrupole moment, corresponding to a deformation of $\left\langle\beta_{2}\right\rangle\left({ }^{45 \mathrm{~g}} \mathrm{Sc}\right)=$ 
$-0.154(7)$. From these values and equation (11), assuming $\left\langle\beta_{2}^{2}\right\rangle=\left\langle\beta_{2}\right\rangle^{2}$, the estimated change in ms charge radii, $\delta\left\langle r^{2}\right\rangle^{45 g, \mathrm{~m}}=+0.43(20) \mathrm{fm}^{2}$, is higher than the experimental value of $\delta\left\langle r^{2}\right\rangle^{45 \mathrm{~g}, \mathrm{~m}}=+0.186(27) \mathrm{fm}^{2}$. This is a striking feature which remains unexplained.

While both $B(\mathrm{E} 2)$ and $\delta\left\langle r^{2}\right\rangle$ measurements have a dependence on the deformation in mean-square form, $\left\langle\beta_{2}^{2}\right\rangle$, the spectroscopic quadrupole moment is dependent on $\left\langle\beta_{2}\right\rangle$. The discrepancy between $\left\langle\beta_{2}^{2}\right\rangle$ and $\left\langle\beta_{2}\right\rangle^{2}$ is often used to infer $\beta$-softness [29], since only the former contains information on the dynamic as well as static components of the deformation. However, in this case, the rms deformation appears less than the mean deformation for the isomeric state. This indicates a breakdown of the collective rotational model on which equation (13) is based. Nevertheless, from a direct comparison of the quadrupole moments, the $Q_{\mathrm{s}}$-derived value of $Q_{0}\left({ }^{45 \mathrm{~m}} \mathrm{Sc}\right)=+1.38(27)$ b is twice as large as $Q_{0}\left({ }^{45 \mathrm{~m}} \mathrm{Sc}\right)=0.746(30) \mathrm{b}$, the average calculated from the $B(\mathrm{E} 2)$ values [13].

\subsubsection{Nuclear radii trends in the neutron $f_{7 / 2}$ shell.}

The present investigation of nuclear radii changes along the scandium isotopic chain complements the published results $[6,7,9]$ on nuclear radii behaviour in the $f_{7 / 2}$ shell. This is illustrated in figures 5 and 6 where the absolute rms nuclear radii values, $R=\left\langle r^{2}\right\rangle^{1 / 2}$, are presented in terms of $N$ and $Z$, respectively. The $R$ values for the $\mathrm{Ar}, \mathrm{K}, \mathrm{Ca}$, $\mathrm{Ti}$ and $\mathrm{Cr}$ isotopes are taken from the recently published consistent sets of rms radii [47] (see also reference [9]). Absolute rms charge radii for the investigated Sc isotopes are derived using as a reference the updated value of $R\left({ }^{45} \mathrm{Sc}\right)=3.5459(25) \mathrm{fm}[48]$ and the error takes into account the model uncertainties.

As can be seen from figure 5 , adding the new data on Sc isotopes to the isotopic radii trend in the $f_{7 / 2}$ shell one obtains a consistent picture: no unreasonable crossing of the isotopic course of the nuclear radii between different elements is observed. More importantly, the shape of the Sc curve shows a steady increase towards $N=20$ similar to $\mathrm{Ti}$ and unlike that of $\mathrm{Ca}$. This is also in contrast with the lower- $Z$ elements Ar and $\mathrm{K}$ for which the radii decrease towards $N=20$. The odd-even staggering along the Sc isotopic curve - especially on the neutron deficient side - is essentially smaller than for the even $-Z$ neighbours of Sc, as is the case for $\mathrm{K}$ (see e.g. reference [6]).

The isotonic trend of nuclear charge radii displayed in figure 6 refers to the even neutron numbers. Unfortunately, the isotonic curves cover the whole $Z$ region from $\mathrm{Ar}$ to Ti only for $N=22$ and $N=24$. A normal proton odd-even staggering effect is observed for $\mathrm{K}$ at $Z=19$ and for $\mathrm{Sc}$ at $Z=21$. This effect is associated with the reduction of core polarization due to unpaired protons.

As shown and discussed in reference [6], a suitable approach for describing the behaviour of the charge radii across the $f_{7 / 2}$ shell is the Zamick-Talmi formula [39, 49],

$$
\delta\left\langle r^{2}\right\rangle^{20,20+n}=n C+\frac{n(n-1)}{2} \alpha+\left[\frac{n}{2}\right] \beta,
$$

where $n$ is the number of neutrons above the $N=20$ shell closure and $[n / 2]=n / 2$ for even $n$ and $[n / 2]=(n-1) / 2$ for odd $n$. According to reference [39] the parameter 


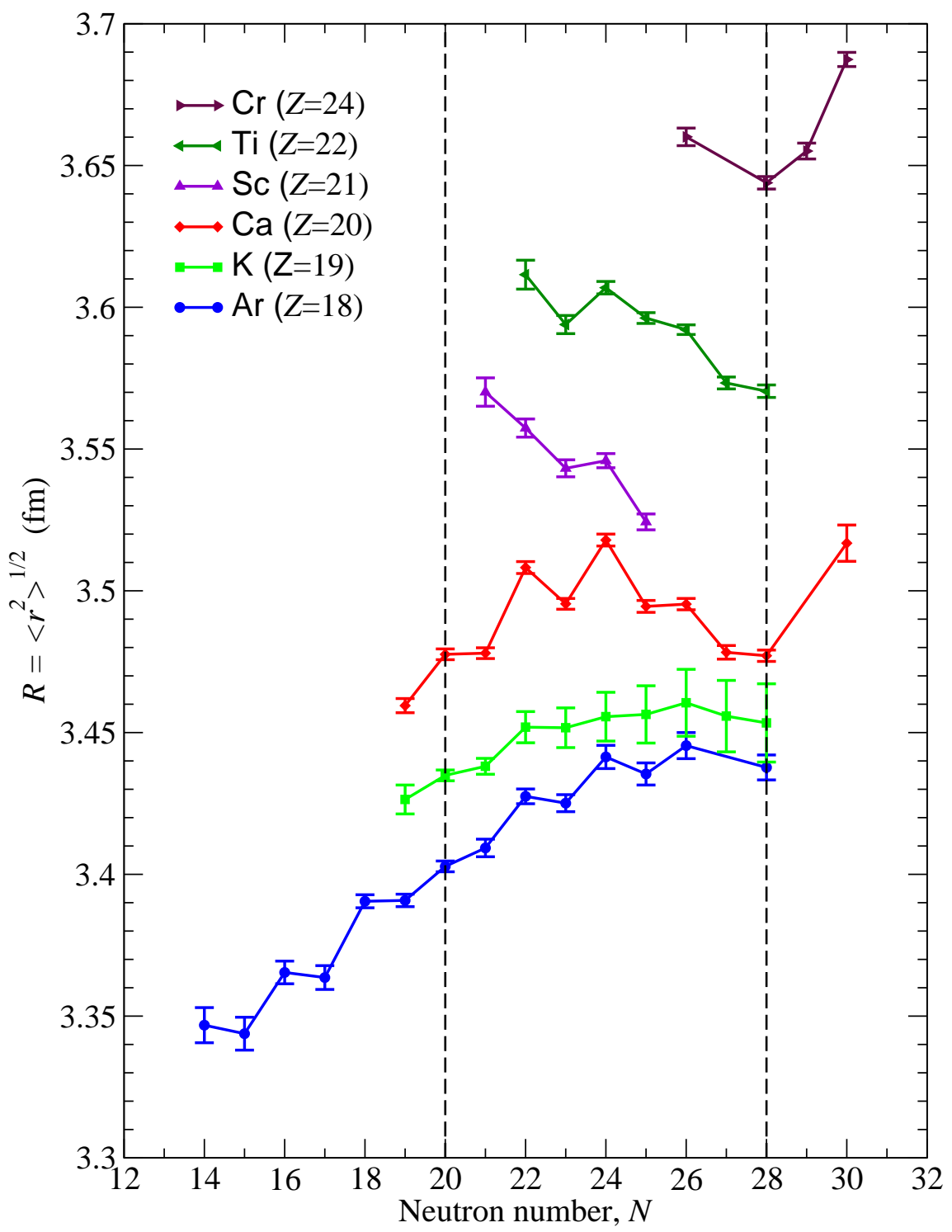

Figure 5. Isotopic dependence of rms nuclear charge radii in the $f_{7 / 2}$ shell.

$C$ expresses the one-body part of the effective charge radius operator, $\alpha$ includes the two-body part and $\beta$ accounts for the odd-even effects. Equation (14) was derived for the ms charge radii of nuclei with only neutrons (or only protons) outside of a closed shell. Nevertheless, it has often been empirically generalised (see eg. reference [50]) and used to fit the trends of radii in other isotope chains. In our case the parameters $C=-0.18(1) \mathrm{fm}^{2}, \alpha=0.015(28) \mathrm{fm}^{2}$ and $\beta=0.16(8) \mathrm{fm}^{2}$ have been determined by a fit to the radii of ${ }^{42-46} \mathrm{Sc}$ and the fitted curve is shown in figure 4 . The Zamick-Talmi formula reproduces well the radii evolution in the case of scandium and thus confirms the general picture of nuclear radii in the $f_{7 / 2}$ shell, including the elements from $Z=18$ to $Z=22$. According to equation (14), the slope of the radii curves is determined by the parameter $C$, connected to the sign of the one-body part of the effective charge radius operator. This parameter is nearly zero for $\mathrm{Ca}$ and changes sign in the transition 


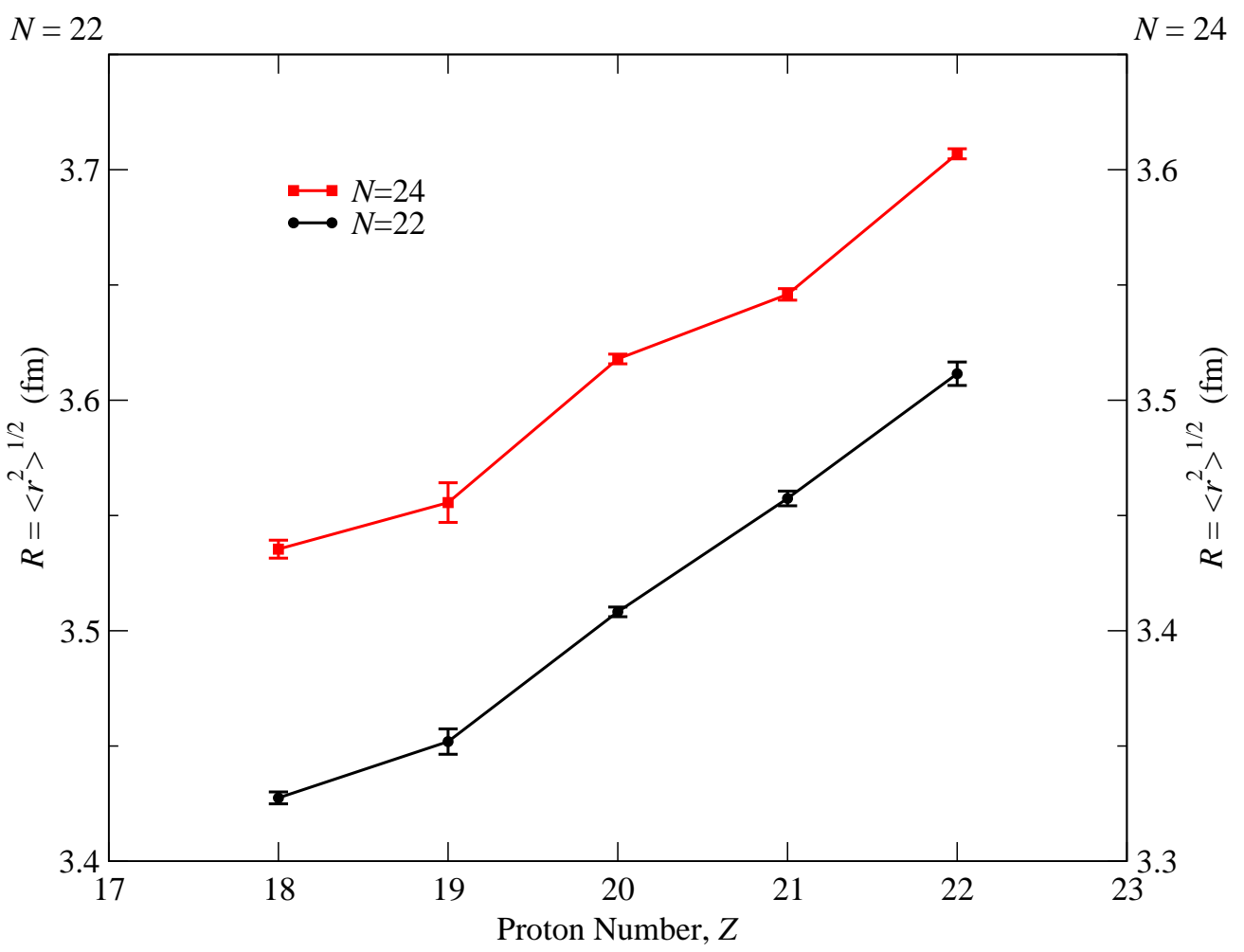

Figure 6. Isotonic dependence of rms nuclear charge radii in the $f_{7 / 2}$ shell.

from $Z<20$ to $Z>20$ (compare our result with those in table 4 of reference [6]). Adding the second and third terms of equation (14) which implicitly contain collective effects like static and dynamic nuclear deformation the trend is changed from a nearly linear dependence to a structure very similar to the experimental one. However, model predictions are necessary for a quantitative description of the observed radii behaviour.

Although there are a large number of theoretical works which deal with different nuclear parameters, including deformation and charge radius, there is no single adequate theoretical approximation explaining even qualitatively the peculiarities of the charge radii trend over the whole $\mathrm{Ca}$ region. The predicted isotopic variation of the charge radii is usually featureless (see e.g. [11, 51]). Several approaches exist which are able to explain some of the observed features. For example, the calculations based on the Hartree-Fock method with Skyrme interactions $[5,6]$ predict the general trend of the Ar charge radii in the $s p$ and $f p$ shell. The shell-model calculations of Caurier et al. [10] using cross-shell proton-neutron correlation are the best description of the charge radii trend for the $\mathrm{Ca}$ isotopes. In the case of $\mathrm{Ti}$ a continual increase in the charge radius going from $N=28$ to the $N=20$ shell closure is qualitatively predicted by the selfconsistent RMF approach $[11,52]$. However, the RMF theory makes no predictions for odd $-Z$ or odd $-N$ nuclei, thus providing no information on the pronounced odd-even effects in $N$ and $Z$.

Once again we note that the isotopic behaviour in the $\nu f_{7 / 2}$ shell around the proton shell closure $Z=20$ is very unusual compared with the data collected for the shell 
closures $N=50,82$ and 126 in the neighbourhood of $Z=50$ and 82 [9]. In the latter cases the overall slope of the isotopic curves as well as the neutron shell-effect (kinks at the magic neutron number) are nearly unaffected by the proton number. This is already pointed out in reference [6] assuming that the different nature of the shell closures and the proton number dependence of the neutron shell gaps for $N=20$ and $N=28$ in the neighbourhood of $Z=20$ are responsible for such an exceptional situation. This is so far an open question and a challenge for the theory.

4.1.3. Theoretical estimates. Table 4 and figure 7 report shell-model results for the shifts of all the Sc isotopes considered here. Following reference [10], we performed unrestricted diagonalizations in the $\left(s_{1 / 2}, d_{3 / 2}, f_{7 / 2}, p_{3 / 2}\right)$ model-space using the ANTOINE code $[53,54]$ and employed the "zbm2.renorm" interaction from the code's package [55]. The shifts were determined by comparing the occupations of protons promoted to the $f p$ shell by correlation effects and assuming harmonic oscillator wave functions. A constant oscillator length of $b=1.974 \mathrm{fm}$ was taken as an average of the optimal values of all the isotopes.

We have checked that the present calculations reproduce well the Ca shifts of reference [10], as shown in the lower panel of figure 7 . In spite of this, the results are noticeably worse for Sc isotopes, with the measured charge radii decreasing with the neutron number and the overall trend not being reproduced. The relative shifts with respect to ${ }^{45 \mathrm{~g}} \mathrm{Sc}$ are correct for ${ }^{45 \mathrm{~m}} \mathrm{Sc}$ and the two ${ }^{44} \mathrm{Sc},{ }^{44 \mathrm{~m}} \mathrm{Sc}$. As one can see from figure 7 the magnitude of the shifts, whether having the correct sign or not, is always underestimated, with the only exception of ${ }^{44} \mathrm{Sc}$. This suggests that the zbm2.renorm interaction does not lower the gap at $Z=20$ properly for Sc and so underestimates the amount of excitations across the $s d$ and $p f$ shells.

\subsection{Nuclear moments}

To better understand the behaviour of the zbm2.renorm interaction, we calculated the magnetic and quadrupole moments in both a $0 \hbar \omega$ model-space (i.e. with no cross shell excitation allowed) and the fully unrestricted model-space, listed in tables 5 and 6. For the full model-space case, the predicted quadrupole moments deviate largely from the experiment and show that one misses a correct description of deformation and charge radii even when all admixtures of $n \mathrm{p}-n \mathrm{~h}$ are allowed. We note that the predicted quadrupole moments are sensibly better for the $0 \hbar \omega$ calculation. This is not unexpected since the zbm2 interaction is based on the SDPF-NR one, which was originally developed to be used only in model-spaces without excitations of nucleons from the $s d$ to the $f p$ orbits. Some modifications needed to apply the zbm2 interaction in the unrestricted space are discussed in reference [56] taking rotational bands as an example. There, it is argued that these lead to a degradation of the quality of quadrupole moments, in accordance with our finding in tables 5 and 6 . On the other hand, full cross shell excitation are an essential mechanism underlying isotope shifts and need to be considered 


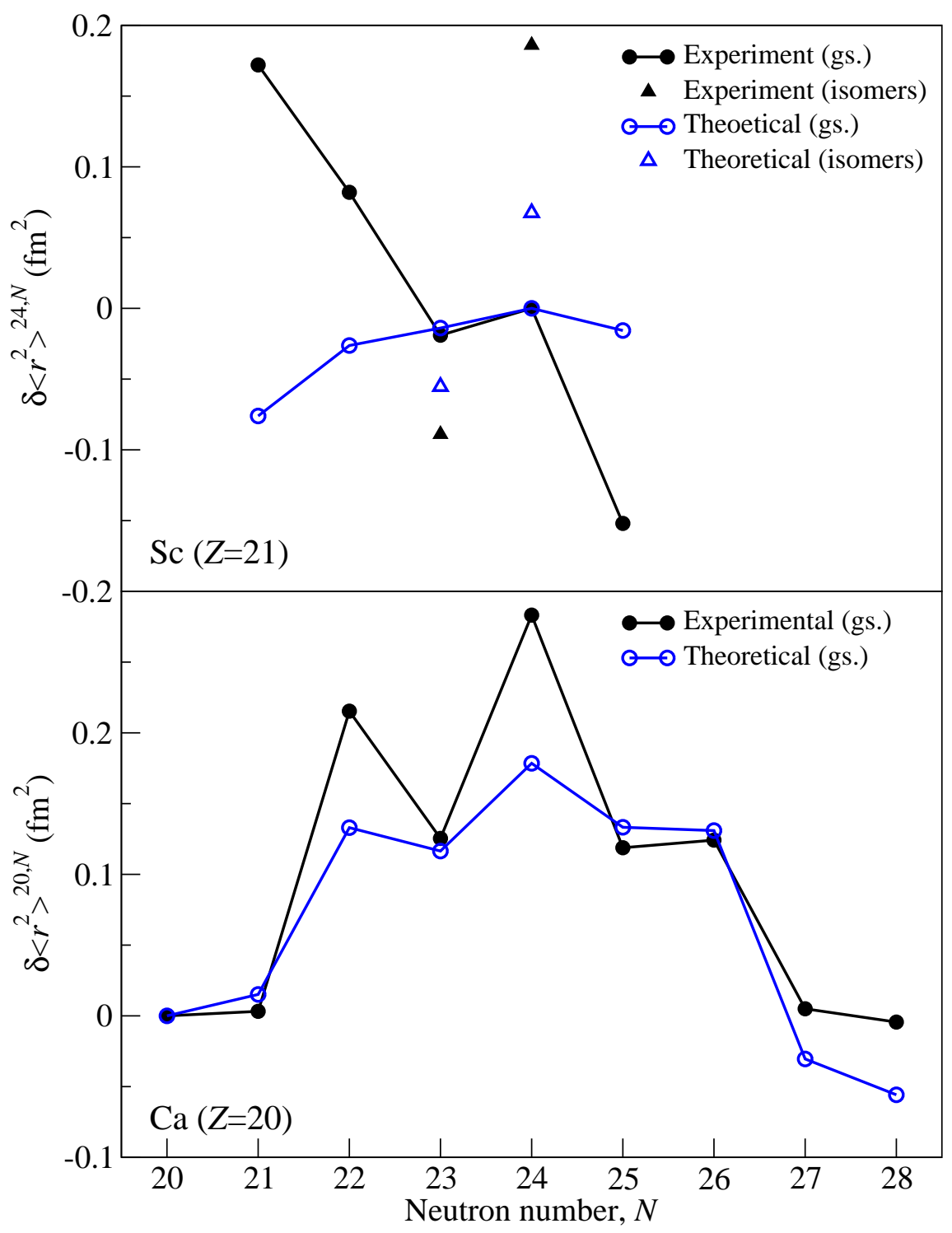

Figure 7. Comparison of experimental $\mathrm{Ca}$ and $\mathrm{Sc}$ ms charge radii with theoretical estimates performed as part of this work.

in the present case [10].

It remains clear that the development of effective interactions appropriate to study excitation across the $s d$ and $p f$ shells is still an open question in shell-model theory [58]. In order to understand properly the shifts in the Sc region one might require further improvements of the SDPF or zbm2 interactions but this is beyond the scope of the present investigation.

The electromagnetic moments of the ${ }^{43-46,44 \mathrm{~m}} \mathrm{Sc}$ isotopes have also been discussed within the framework of shell-model calculations for the $f p$ shell by van der Merwe et al. [57]. Their model assumes a ${ }^{40} \mathrm{Ca}$ core and a model-space which consists of configurations $0 f_{7 / 2}^{n}+0 f_{7 / 2}^{n-1}\left(1 p_{3 / 2} 0 f_{5 / 2} 1 p_{1 / 2}\right)^{1}$. Effective $g$-factors $\left(g_{\pi}^{s}=+5.031\right.$, $g_{\nu}^{s}=-3.041, g_{\pi}^{l}=+1.000$ and $\left.g_{\nu}^{l}=0\right)$ and effective charges $\left(e_{\pi}=+1.486\right.$ and 
Table 5. Nuclear magnetic dipole moments, $\mu$, obtained in this work (calibrated using the value for ${ }^{45} \mathrm{Sc}[15]$ ) compared with shell-model predictions of this work (using the same interaction employed for calculating the isotope shifts and the effective $g$-factors $\left.g_{l}^{\pi}=+1, g_{l}^{\nu}=0, g_{s}^{\pi}=+5.031, g_{s}^{\nu}=-3.041\right)$ and calculations of reference [57].

\begin{tabular}{llllll}
\hline$A$ & $I^{\pi}$ & $\begin{array}{l}\mu\left(\mu_{\mathrm{N}}\right) \\
(\text { experimental })\end{array}$ & $\begin{array}{l}\mu\left(\mu_{\mathrm{N}}\right) \\
(\text { theoretical }[57])\end{array}$ & $\begin{array}{l}\mu\left(\mu_{\mathrm{N}}\right) \\
(0 \hbar \omega)\end{array}$ & $\begin{array}{l}\mu\left(\mu_{\mathrm{N}}\right) \\
(\text { unrestricted space) }\end{array}$ \\
\hline 43 & $7 / 2^{-}$ & $+4.528(10)$ & +4.687 & +0.491 & +4.403 \\
44 & $2^{+}$ & $+2.499(5)$ & +2.532 & +2.585 & +1.435 \\
$44 \mathrm{~m}$ & $6^{+}$ & $+3.833(12)$ & +3.831 & +4.081 & +3.597 \\
45 & $7 / 2^{-}$ & $+4.756487(2)^{\dagger}$ & +4.728 & +4.390 & +4.878 \\
$45 \mathrm{~m}$ & $3 / 2^{+}$ & $+0.360(11)$ & - & - & +0.297 \\
46 & $4^{+}$ & $+3.042(8)$ & +2.974 & +3.263 & +2.266 \\
\hline
\end{tabular}

$\dagger$ reference value [15]

Table 6. Nuclear spectroscopic quadrupole moments, $Q_{\mathrm{s}}$, obtained in this work (calibrated using the value for ${ }^{45} \mathrm{Sc}$ [15]) compared with shell-model predictions of this work (using the same interaction employed for calculating the isotope shifts and the effective charges $\left.e_{\pi}=+1.486, e_{\nu}=+0.840\right)$ and calculations of reference [57].

\begin{tabular}{llllll}
\hline$A$ & $I^{\pi}$ & $\begin{array}{l}Q_{\mathrm{s}}(\mathrm{b}) \\
(\text { experimental) }\end{array}$ & $\begin{array}{l}Q_{\mathrm{s}}(\mathrm{b}) \\
\text { (theoretical [57]) }\end{array}$ & $\begin{array}{l}Q_{\mathrm{s}}(\mathrm{b}) \\
(0 \hbar \omega)\end{array}$ & $\begin{array}{l}Q_{\mathrm{s}}(\mathrm{b}) \\
\text { (unrestricted space) }\end{array}$ \\
\hline 43 & $7 / 2^{-}$ & $-0.27(5)$ & -0.2300 & -0.1937 & -0.1534 \\
44 & $2^{+}$ & $+0.16(4)$ & +0.0545 & +0.0322 & -0.0664 \\
$44 \mathrm{~m}$ & $6^{+}$ & $-0.21(9)$ & -0.2648 & -0.2378 & -0.0399 \\
45 & $7 / 2^{-}$ & $-0.220(2)^{\dagger}$ & -0.2550 & -0.1948 & -0.1198 \\
$45 \mathrm{~m}$ & $3 / 2^{+}$ & $+0.28(5)$ & - & - & +0.2055 \\
46 & $4^{+}$ & $+0.12(2)$ & -0.0246 & -0.0363 & -0.0757 \\
\hline
\end{tabular}

$\dagger$ reference value [15]

$\left.e_{\nu}=+0.840\right)$ were used in reference [57] to compensate for the configurations excluded by the model-space. The calculated electromagnetic moments are listed in tables 5 and 6 and it can be seen that the predictive power of these calculations is excellent for the magnetic moments. The experimental value of $\mu\left({ }^{45 \mathrm{~m} S c}\right)=+0.360(11) \mu_{\mathrm{N}}$ lies well above the single-particle magnetic moment $+0.126 \mu_{\mathrm{N}}$ of the $d_{3 / 2}$ proton and reflects the mixed structure of this isomeric state.

Conclusions from a comparison between theory and experiment are more qualitative for the quadrupole moments than for the magnetic moments. There are two reasons for this: (i) the E2 matrix elements are more complicated than those for the M1 operator, because they require explicitly the radial wave function, and (ii) the experimental values of the quadrupole moments have larger errors, and often the absolute calibration, depending on the atomic properties, is subject to uncertainties of about $10 \%$. For ${ }^{46} \mathrm{Sc}$ the theoretical prediction from reference [57] for the spectroscopic quadrupole moment is close to zero, while in the remaining cases the measured spectroscopic quadrupole moments follow at least the predicted trend of the sign. A close agreement is achieved 
in the case of ${ }^{45} \mathrm{Sc}$, where a spectroscopic quadrupole moment of $Q_{\mathrm{s}}=-0.217 \mathrm{~b}$ is obtained in reference [43] by the modified (kb5) Kuo-Brown interaction. Calculations performed in this work predict a quadrupole moment for the ${ }^{45 \mathrm{~m}} \mathrm{Sc}$ isomer which is in reasonable agreement with the experimental value, although underestimated (as is the ms charge radius).

\section{Conclusion}

Laser spectroscopy has been performed on ${ }^{42,43,44,44 \mathrm{~m}, 45,45 \mathrm{~m}, 46} \mathrm{Sc}$ revealing the nuclear moments and ms charge radii. The latter show an increase with decreasing neutron number and a reduction in odd-even staggering relative to the even- $Z$ neighbouring chains. Shell-model calculations were performed and a comparison with the experimental values highlights the need to improve the effective interaction used.

The data obtained in the present work complement the nuclear radii systematics in the $f_{7 / 2}$ shell for elements around $Z=20$. However, to achieve a full picture of the nuclear properties in this $(Z, N)$ region the experimental information needs to be extended still further. Of great importance is the continuation of the optical investigations of $\mathrm{Sc}$ and $\mathrm{Ti}$ isotopes toward and beyond the shell closures at $N=20$ and $N=28$. Information obtained on nuclear radii and moments for isotopes far from stability and around neutron magic number provide a stringent test of the available theoretical models.

\section{Acknowledgements}

This work has been supported by a Joint Project Grant from the Royal Society, the UK Engineering and Physical Sciences Research Council, the Science and Technology Facilities Council, the Russian Foundation for Basic Research grant 04-02-16955 and the Academy of Finland under the Finnish Centre of Excellence Programme 2000-2005 (project No 44875). SF acknowledges support by the FiDiPro programme of the Finnish Academy. We are grateful to Dr. P. Bednarczyk for the fruitful discussion of the nuclear quadrupole moments.

\section{References}

[1] Palmer C W et al 1984 J. Phys. B 172197

[2] Vermeeren L et al 1992 Phys. Rev. Lett 681679

[3] Vermeeren L et al 1996 J. Phys. G: Nucl. Part. Phys. 221517

[4] Touchard F et al 1982 Phys. Lett. B 108169

[5] Klein A et al 1996 Nucl. Phys. A 6071

[6] Blaum K et al 2008 Nucl. Phys. A $\mathbf{7 9 9} 30$

[7] Gangrsky Y P et al 2004 J. Phys. G: Nucl. Part. Phys. 301089

[8] Otten E W 1989 in Treatise on Heavy-Ion Science, edited by Bromley D A (NY: Plenum Press), vol 8, p 517

[9] Angeli I et al 2009 J. Phys. G: Nucl. Part. Phys. 36085102

[10] Caurier E et al 2001 Phys. Lett. B $\mathbf{5 2 2} 240$

[11] Lalazissis G A, Raman S and Ring P 1999 At. Data Nucl. Data Tables 711 
[12] Cheal B and Flanagan K T 2010 J. Phys. G: Nucl. Part. Phys. 37113101

[13] Bednarczyk P et al 1998 Eur. Phys. J. A 2157

[14] Bednarczyk P et al 2004 Eur. Phys. J. A 2045

[15] Stone N J 2005 At. Data Nucl. Data Tables 9075

[16] Gangrsky Y et al 2006 Hyperfine Interact. 171209

[17] Äystö J 2001 Nucl. Phys. A 693477

[18] Nieminen A et al 2001 Nucl. Instr. and Meth. A 469244

[19] Nieminen A et al 2002 Phys. Rev. Lett. 88094801

[20] Campbell P et al 2002 Phys. Rev. Lett. 89082501

[21] Kopfermann H 1958 Nuclear Moments (NY: Academic Press Inc)

[22] Büttgenbach S 1984 Hyperfine Interact. 201

[23] King W H 1984 Isotope shifts in atomic spectra (Plenum Press)

[24] Zimmermann D et al 1980 Z. Phys. A 295307

[25] Blundell S et al 1985 Z. Phys. A 32131

[26] Blundell S A et al 1987 J. Phys. B: At. Mol. Opt. Phys. 203663

[27] Fischer W, Hühnermann H, Krömer G and Schäfer H 1974 Z. Phys. 270113

[28] Cheal B et al 2009 Phys. Rev. Lett. 102222501

[29] Cheal B et al 2007 Phys. Lett. B 645133

[30] Fischer A et al 2010 Phys. Rev. Lett. 104073004

[31] Fritzsche S 2002 Phys. Scr. T100 37

[32] Grant I P 1988 in Methods in Computational Chemistry, edited by Wilson S (NY: Plenum Press), vol 2, p 1

[33] Parpia F A, Froese Fischer C and Grant I P 1996 Comput. Phys. Commun. 94249

[34] Fritzsche S 2001 J. Electron Spectrosc. Relat. Phenom. 114-116 1155

[35] Gaigalas G, Fritzsche S and Grant I P 2001 Comput. Phys. Commun. 139263

[36] Fritzsche S, Froese Fischer C and Gaigalas G 2002 Comput. Phys. Commun. 148103

[37] Mårtensson-Pendrill A M et al 1992 Phys. Rev. A 454675

[38] Sewtz M et al 2003 Phys. Rev. Lett. 90163002

[39] Zamick L 1971 Ann. Phys. 66784

[40] Styczen J et al 1976 Nucl. Phys. A 262317

[41] Bednarczyk P et al 1997 Phys. Lett. B 393285

[42] Bednarczyk P et al 2001 Acta Phys. Pol. B 32747

[43] Cole B J 1985 J. Phys. G: Nucl. Part. Phys. 11953

[44] Poves A and Solano J S 1998 Phys. Rev. C 58179

[45] Myers W D and Schmidt K H 1983 Nucl. Phys. A 41061

[46] Berdichevsky D and Tondeur F 1985 Z. Phys. A: Hadrons Nucl. 322141

[47] Database of the Lomonosov Moscow State University, Skobeltsyn Institute of Nuclear Physics, http://cdfe.sinp.msu.ru/services/radchart/radmain.html

[48] Angeli I 2010 by private communication

[49] Talmi I 1984 Nucl. Phys. A $\mathbf{4 2 3} 189$

[50] Wohlfahrt H D et al 1981 Phys. Rev. C 23533

[51] Goriely S, Tondeur F and Pearson J M 2001 Atomic Data and Nuclear Data Tables 77311

[52] Lalazissis G A, Farhan A R and Sharma M M 1998 Nucl. Phys. A 628221

[53] Caurier E et al 2005 Rev. Mod. Phys. 77427

[54] Caurier E and Nowacki F 1999 Acta Phys. Pol. B 30705

[55] http://sbgat194.in2p3.fr/ theory/antoine/main.html

[56] Poves A, Caurier E, Nowacki F and Zuker A 2004 Eur. Phys. J. A 20119

[57] van der Merwe M G, Richter W A and Brown B A 1994 Nucl. Phys. A 579173

[58] Utsuno Y et al 2009 AIP Conf. Proc. 112081 